\title{
NEW USES OF THE WESTPHAL CONUENSATION. SYNTHESIS OF PI-DONOR-PI-ACCEPTOR HETEROCYCLES.
}

\author{
Maria P. Matia ${ }^{a}$, Jesús Ezquerrab, Francisco Sánchez- \\ Ferrandoc, José L. Garcia-Navio ${ }^{a}$, Juan J. Vaquero ${ }^{a}$ \\ and Julio Alvarez-Builla*a. \\ a Departamento de Quimica orgánica, Universidad de \\ Alcalá, 28871 Alcalá de Henares, Madrid (Spain) \\ b Centro de Investigacion LILLY S.A., Paraje de la Cruz \\ $s / n, 28130$ Valdeolmos, Madrid (Spain) \\ c Departament de Quimica, Universitat Autonoma de \\ Barcelona, 08193 Bellaterra, Barcelona (Spain)
}

(Received in UK 29 May 1991)

\begin{abstract}
Novel pyrido[1,2-a] and pyridazino[2, 3-a]pyrrolo[2,1-c]pyrazin-7-ium derivatives have been prepared using Westphal condensation, as an example of fused heterocycles with linked $\pi$-donor and $\pi$-acceptor moieties.
\end{abstract}

Following our interest in dipolar heterocycles, we are exploring the preparation of polycyclic derivatives, in which $\pi$-donor and $\pi$-acceptor moieties are covalently bonded in a planar system, allowing a good transfer of electronic density between them.

As one of the more evident examples, we focussed our attention on compounds with a 2,2'-pyrrolylpyrazinium substructure 1 ike pyrido[1,2-a]pyrrolo[2,1-c]pyrazin-7-ium salts $1\left(\mathrm{R}_{1}, \mathrm{R}_{2}=\mathrm{H}, \mathrm{A}-\mathrm{B}=-\mathrm{CH}=\mathrm{CH}-\right)$ (Fig. 1$)$, in which the pyrrole moiety would increase the contribution of the resonance form II to the electronic distribution of the ground state.<smiles>[Y]n1cccc1-c1cccc[n+]1B</smiles>

1<smiles></smiles>

$\|$

Fig. 1 
The positive charge of the nucleus would be efficiently delocalized between both nitrogen atoms, producing comparatively stable structures of unusual reactivity and physico-chemical properties. One related example has been described by K. Matobal, obtained by a non generalizable process.

After previous contributions ${ }^{2-6}$ to Westphal condensation ${ }^{7}$, we have designed a general route for derivatives type 1 by starting with 1methyl-3,4-dihydropyrrolo[1,2-a]pyrazine ${ }^{8}\left(\mathrm{R}_{1}, \mathrm{R}_{2}=\mathrm{H}, \mathrm{A}-\mathrm{B}=-\mathrm{CH}_{2}-\mathrm{CH}_{2}-\right), 1-$ methylpyrrolo[1,2-a]pyrazine ${ }^{9}\left(\mathrm{R}_{1}, \mathrm{R}_{2}=\mathrm{H}, \mathrm{A}-\mathrm{B}=-\mathrm{CH}=\mathrm{CH}-\right)$, and 1-methyl-3,4dihydropyrazino[1,2-a] indole $\left(\mathrm{R}_{1}, \mathrm{R}_{2}=-(\mathrm{CH}=\mathrm{CH}) 2^{-}, \mathrm{A}-\mathrm{B}=-\mathrm{CH}_{2}-\mathrm{CH}_{2}^{-}\right)$.

When these heterocycles were treated with ethyl bromoacetate the quaternary salts $\mathbf{2}, \mathbf{3}$ and $\mathbf{4}$ were obtained (Fig. 2). However, quaternization of 1 -methyl-6H-pyrrolo[1,2-a]quinoxaline ${ }^{10}\left(R_{1}, R_{2}=H, A-B=\right.$ 1,2-phenylene) was unsuccessful.

Basic condensation of bromides 2-3 with 1,2-dicarbonyl derivatives yielded the related pyrido $[1,2-a]$ pyrrolo $[2,1-c]$ pyrazin-7-ium salts 9 -10 and the 6,7 -dihydropyrido $\left[2^{\prime}, 1^{\prime}: 3,4\right]$ pyrazino $[1,2$-a] indo1-8-ium salt 11 (Fig. 2). As in previous papers ${ }^{3-5}$, three representative 1,2 -dicarbonyls were used to test the applicability of the method, producing all of them condensation products.

The $\mathrm{N}$-amination of the related heterocyclic precursors produced the derivatives 5-8, which were used in a modified Westphal process ${ }^{6}$, to generate the novel salts 12-14, aza analogues of the bromides 9-11, and the pyrrolo[1,2-a]pyridazino[3,2-c]quinoxalin-9-ium mesitylenesulfonates 15 (Fig.2). As it has been previously observed 6 , the reaction was produced only with polycyclic o-quinones, while benzil and diacetyl were recovered untransformed. All new compounds obtained are listed in Table 1. 

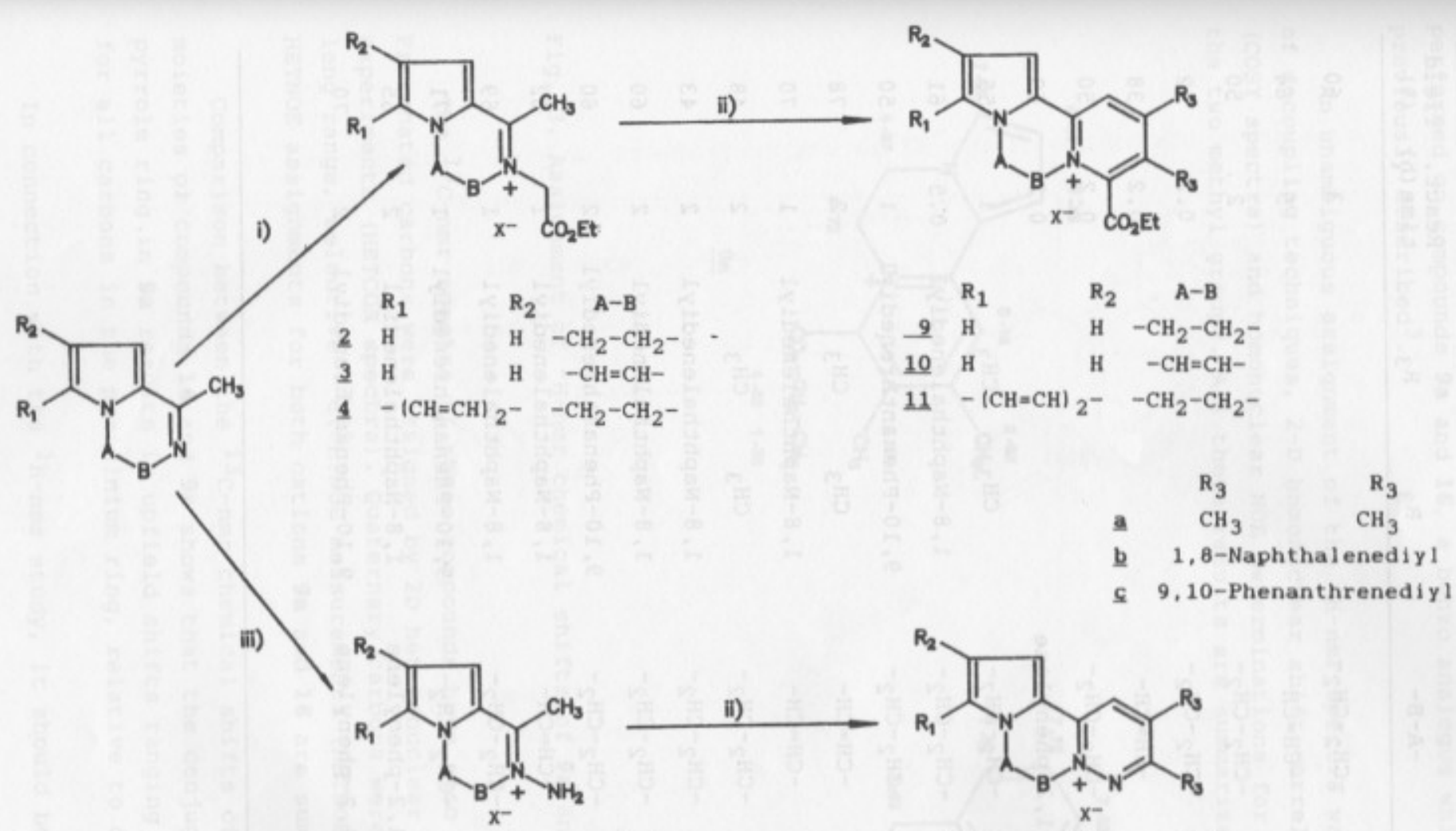

$\begin{array}{llcc} & \mathrm{R}_{1} & \mathrm{R}_{2} & \mathrm{~A}-\mathrm{B} \\ \mathbf{5} & \mathrm{H} & \mathrm{H} & -\mathrm{CH}_{2}-\mathrm{CH}_{2}^{-} \\ \underline{6} & \mathrm{H} & \mathrm{H} & -\mathrm{CH}=\mathrm{CH}^{-} \\ \underline{7} & -(\mathrm{CH}=\mathrm{CH}) & 2^{-} & -\mathrm{CH}_{2}-\mathrm{CH}_{2}^{-} \\ \underline{8} & \mathrm{H} & \mathrm{H} & 1,2-\text { phenylene }\end{array}$
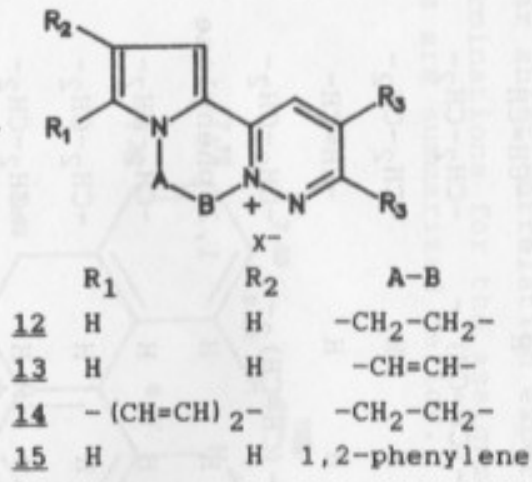

Fig 2

1) $\mathrm{BrCH}_{2} \mathrm{CO}_{2} \mathrm{Et} /$ Acetone, $\triangle$

ii) $\mathrm{R}_{3} \mathrm{COCOR}_{3} / \mathrm{Na}^{+}, \mathrm{CH}_{3} \mathrm{CO}_{2}{ }^{-} / /$Acetone, $\Delta$

1111 o-Hydroxylaminomesitylenesulfonate/ether, r.t. 
Table 1. New derivatives prepared

\begin{tabular}{|c|c|c|c|c|c|c|}
\hline $\begin{array}{l}\text { Compd. } \\
\text { No. }\end{array}$ & $\cdot \mathrm{R}_{1}$ & $\mathrm{R}_{2}$ & $-\mathrm{A}-\mathrm{B}-$ & $\mathrm{R}_{3}$ & $\begin{array}{l}\text { React. } \\
\text { time (h) }\end{array}$ & $\begin{array}{c}\text { Yield } \\
\text { (8) }\end{array}$ \\
\hline 2 & H & $\mathrm{H}$ & $-\mathrm{CH}_{2}-\mathrm{CH}_{2}-$ & & 3 & 60 \\
\hline 3 & H & $\mathrm{H}$ & $-\mathrm{CH}=\mathrm{CH}-$ & & 5 & 64 \\
\hline 4 & $-1 \mathrm{CH}$ & H) $2^{-}$ & $-\mathrm{CH}_{2}-\mathrm{CH}_{2}^{-}$ & & 2 & 50 \\
\hline 5 & H & $\mathrm{H}$ & $-\mathrm{CH}_{2}-\mathrm{CH}_{2}^{-}$ & & 0.2 & 52 \\
\hline 6 & $\mathrm{H}$ & $\mathrm{H}$ & $-\mathrm{CH}=\mathrm{CH}-$ & & 0.2 & 38 \\
\hline 7 & $-(\mathrm{CH}$ & H) $2^{-}$ & $-\mathrm{CH}_{2}-\mathrm{CH}_{2}^{-}$ & & 0.2 & 50 \\
\hline 8 & $\mathrm{H}$ & $\mathrm{H}$ & 1,2 -phenylene & & 0.2 & 63 \\
\hline $9 a$ & H & H & $-\mathrm{CH}_{2}-\mathrm{CH}_{2}^{-}$ & $\mathrm{CH}_{3}$ & 1 & 55 \\
\hline $9 \mathrm{~b}$ & $\mathrm{H}$ & $\mathrm{H}$ & $-\mathrm{CH}_{2}-\mathrm{CH}_{2}-$ & 1,8-Naphthalenediyl & 0.5 & 61 \\
\hline $9 c$ & $\mathrm{H}$ & $\mathrm{H}$ & $-\mathrm{CH}_{2}-\mathrm{CH}_{2}^{-}$ & 9,10 -Phenanthrenediy 1 & 1 & 50 \\
\hline $10 \mathrm{a}$ & $\mathrm{H}$ & $\mathrm{H}$ & $-\mathrm{CH}=\mathrm{CH}-$ & $\mathrm{CH}_{3}$ & 2 & 78 \\
\hline $10 \mathrm{~b}$ & $\mathrm{H}$ & $\mathrm{H}$ & $-\mathrm{CH}=\mathrm{CH}-$ & 1,8 -Naphthalenediy 1 & 1 & 70 \\
\hline $11 \mathbf{a}$ & $-1 \mathrm{CH}$ & $2^{-}$ & $-\mathrm{CH}_{2}-\mathrm{CH}_{2}^{-}$ & $\mathrm{CH}_{3}$ & 2 & 48 \\
\hline $11 b$ & $-(\mathrm{CH}$ & н) $2^{-}$ & $-\mathrm{CH}_{2}-\mathrm{CH}_{2}^{-}$ & 1,8-Naphthalenediy 1 & 2 & 43 \\
\hline $12 \mathrm{~b}$ & H & $\mathrm{H}$ & $-\mathrm{CH}_{2}-\mathrm{CH}_{2}-$ & 1,8 -Naphthalenediyl & 2 & 60 \\
\hline $12 c$ & $\mathrm{H}$ & $\mathrm{H}$ & $-\mathrm{CH}_{2}-\mathrm{CH}_{2}^{-}$ & 9,10 -Phenanthrenediy 1 & 2 & 60 \\
\hline $13 \mathrm{~b}$ & $\mathrm{H}$ & $\mathrm{H}$ & $-\mathrm{CH}=\mathrm{CH}-$ & $1,8-$ Naphthalenediy 1 & 1 & 62 \\
\hline $14 \mathrm{~b}$ & $-(\mathrm{CH}$ & $2^{-}$ & $-\mathrm{CH}_{2}-\mathrm{CH}_{2}-$ & 1,8 -Naphthalenediy 1 & 1 & 69 \\
\hline $14 \mathrm{c}$ & $-(\mathrm{CH}$ & H) $2^{-}$ & $-\mathrm{CH}_{2}-\mathrm{CH}_{2}-$ & 9,10-Phenanthrenediy 1 & 1 & 71 \\
\hline $15 b$ & H & $\mathrm{H}$ & 1,2 -phenylene & 1,8-Naphthalenediy 1 & 2 & 65 \\
\hline $15 c$ & $\mathrm{H}$ & $\mathrm{H}$ & 1,2-phenylene & 9,10 -Phenanthrenediy 1 & 2 & 70 \\
\hline
\end{tabular}


As a way to establish if the contribution of the form II was significant to the resonance hybrid, a completely ${ }^{1} \mathrm{H}$ and ${ }^{13} \mathrm{C}-\mathrm{nm} r$ study was performed on compounds $9 \mathrm{a}$ and 16 , a benzo analogue which has been previously described ${ }^{3}$.

An unambiguous assignment of the ${ }^{1} \mathrm{H}-\mathrm{nmr}$ spectra was achieved by means of decoupling techniques, 2-D homonuclear shift correlation experiments (COSY spectra) and homonuclear NOE determinations for the assignment of the two methyl groups. All these results are summarized bellow.

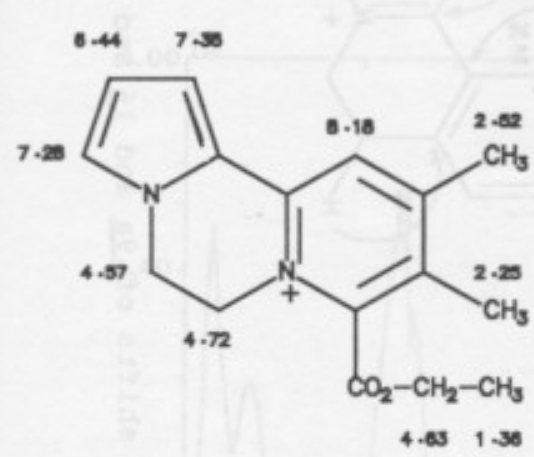

$\underline{9 a}$

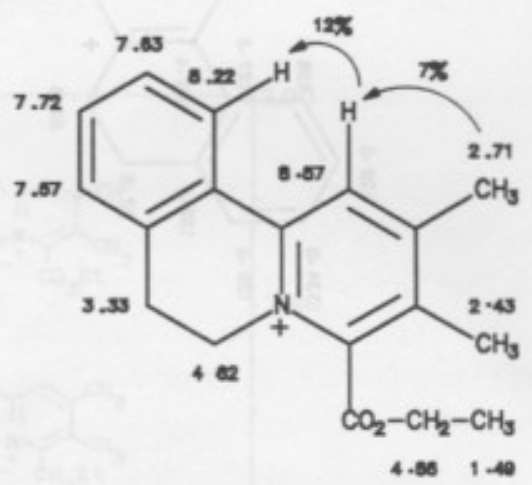

$\underline{16}$

Fig. 3. Assignment of ${ }^{1} \mathrm{H}-\mathrm{nmr}$ chemical shifts of $9 \mathbf{a}$ and 16 .

The ${ }^{13} \mathrm{C}-\mathrm{nmr}$ spectra of these compounds have also been determined. Protonated carbons were assigned by 2D heteronuclear shift correlation experiments (HETCOR spectra). Quaternary carbons were assigned using long range, selective $\left.{ }^{13} \mathrm{C} f^{1} \mathrm{H}\right\} \mathrm{NOE}$ measurements (HETNOE method) ${ }^{11,12}$. The HETNOE assignments for both cations $9 \mathrm{a}$ and 16 are summarized in Fig. 4 .

Comparison between the ${ }^{13} \mathrm{C}-\mathrm{nmr}$ chemical shifts of the pyridinium moieties of compounds 16 and 9 a shows that the conjugation with the pyrrole ring in 9 a results in upfield shifts ranging from 2.0 to $5.0 \mathrm{ppm}$ for all carbons in the pyridinium ring, relative to compound 16.

In connection with the ${ }^{1} \mathrm{H}-\mathrm{nm} r$ study, it should be pointed out that 


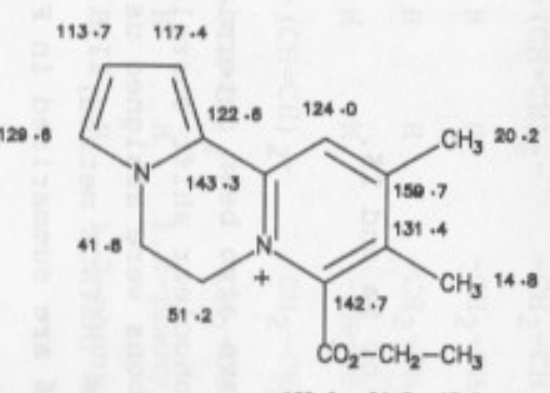

9 a

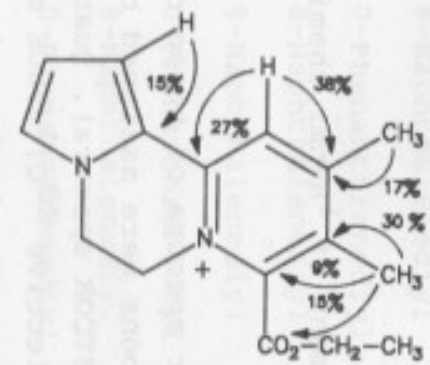

$9 \mathrm{a}$
120.0

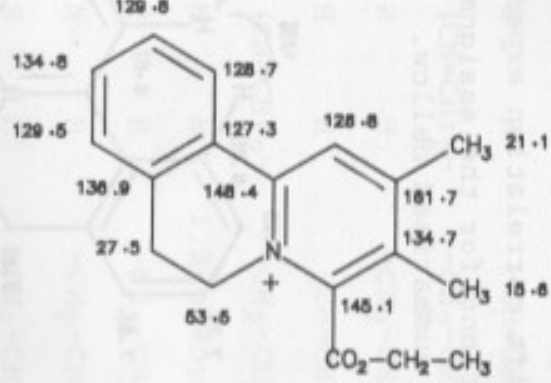

16

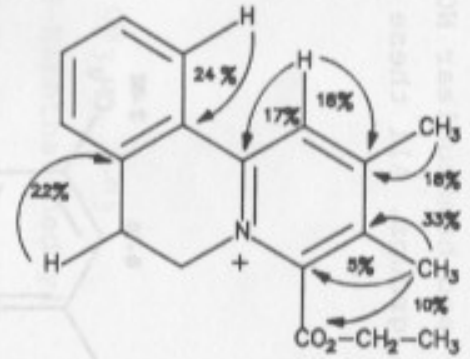

16

Fig 4. Assignment of ${ }^{13} \mathrm{C}-\mathrm{nm}$ chemical shifts of $\underline{9 a}$ and $\underline{16}$ and HETNOE measurents. 
our results are not in agreement with previous literature assignment ${ }^{8}$, where authors described ${ }^{1} \mathrm{H}$-nmr data for some simple quaternary salts, as 2-methyl and 2-phenethyl 1-methyl-3,4-dihydropyrrolo[1,2-a]pyrazines.

Transfer of electronic density between the pyrrolo and the pyridinium moieties has also been observed in the Uv spectra. As an example, in the 5,6-dihydropyrido $[1,2$-a]pyrrolo $[2,1-c]$ pyrazin-7-ium bromide $9 a$, a bathochromic shift can be observed in comparison with the analogous benzo[a]quinolizinium salt 16 (Fig. 5). A similar effect can be observed from compounds 9 to 15 (see experimental section).

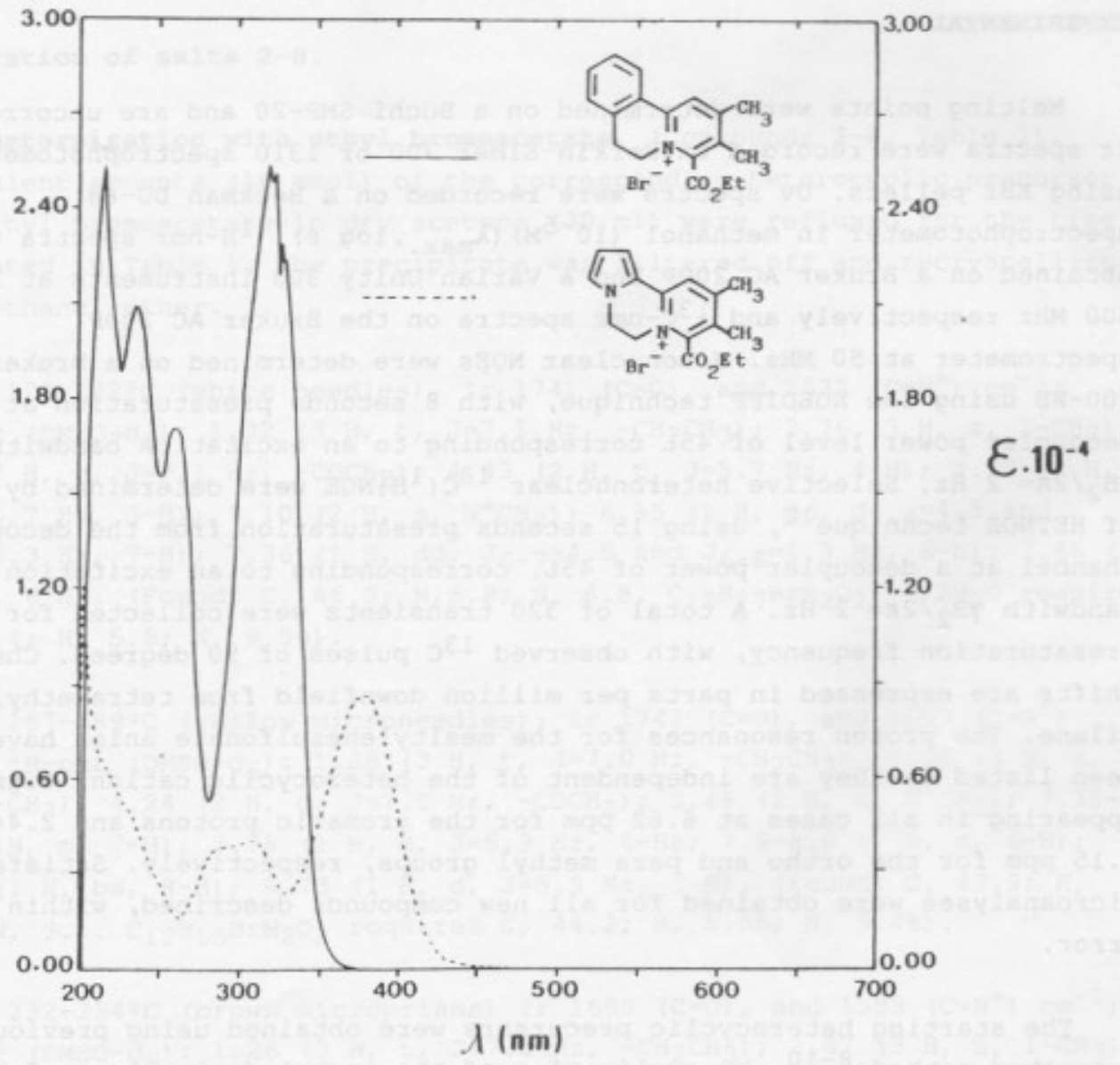

Fig. 5 
As a conclusion, in the pyrido[1,2-a]pyrrolo[2,1-c]pyrazin-7-ium derivative 9 a seems to be a clear transfer of electronic density from the pyrrole to the pyridinium ring, favored by coplanarity in the tricyclic system. This effect appears, with small differences, in all related compounds. Further experiments in relation with the electronic structure of all products are in progress and would be published elsewhere.

In relation with the synthesis, the Westphal condensation provides a simple an efficient methodology for the preparation of fused pyridinium and pyridazinium derivatives. However, the $\mathrm{N}-\mathrm{C}$ condensation ${ }^{6}$ seems rather limited to the use of o-quinones as dielectrophiles.

\section{EXPERTMENTAL}

Melting points were determined on a Büchi SMP-20 and are uncorrected. Ir spectra were recorded on Perkin-Elmer 700 or 1310 spectrophotometers using $\mathrm{KBr}$ pellets. Uv spectra were recorded on a Beckman DU-68 spectrophotometer in methanol $\left(10^{-5} \mathrm{M}\right)\left(\lambda_{\max }, \log \varepsilon\right)$. ${ }^{1} \mathrm{H}-\mathrm{nmr}$ spectra were obtained on a Bruker AC 200P and a Varian Unity 300 instruments at 200 and $300 \mathrm{MHz}$ respectively and ${ }^{13} \mathrm{C}$-nmr spectra on the Bruker $\mathrm{AC} 200 \mathrm{P}$ spectrometer at $50 \mathrm{MHz}$. Homonuclear NOEs were determined on a Bruker AM400-WB using the NOEDIFF technique, with 8 seconds presaturation at a decoupler power level of 45L corresponding to an excitation bandwith $\gamma_{B_{2}} / 2 \pi=2 \mathrm{~Hz}$. Selective heteronuclear $\left.{ }^{13} \mathrm{Cl}^{1} \mathrm{H}\right\} \mathrm{NOE}$ were determined by means of HETNOE technique ${ }^{12}$, using 15 seconds presaturation from the decoupler channel at a decoupler power of $45 \mathrm{~L}$, corresponding to an excitation bandwith $\gamma \mathrm{B}_{2} / 2 \pi=2 \mathrm{~Hz}$. A total of 320 transients were collected for each presaturation frequency, with observed ${ }^{13} \mathrm{C}$ pulses of 90 degrees. Chemical shifts are expressed in parts per million downfield from tetramethylsilane. The proton resonances for the mesitylenesulfonate anion have not been listed as they are independent of the heterocyclic cation, signals appearing in all cases at $6.62 \mathrm{ppm}$ for the aromatic protons and 2.44 and $2.15 \mathrm{ppm}$ for the ortho and para methyl groups, respectively. Satisfactory microanalyses were obtained for all new compounds described, within $0.4 \%$ error.

The starting heterocyclic precursors were obtained using previously described methods ${ }^{8-10}$, by cyclization of the acetyl derivatives of $\mathrm{N}-(2-$ aminoethyl or aminophenyl)pyrrole, and only the indole precursor has not been reported before. The benzo[a]quinolizinium bromide 16 was synthetized ${ }^{3}$ by basic condensation between 1-methy1-2-(ethoxycarbonyl- 
methy1)-3,4-dihydro-isoquinolinium bromide and diacetyl.

1-Methy1-3, 4-dihydropyrazino[1,2-a] indole. N-(2-acetamidoethyl) indole 13 (16.5 mmol) was refluxed for $1 \mathrm{~h}$ in phosphorus oxychloride $(15 \mathrm{ml})$. Then, the mixture was concentrated in vacuo, and the residue was dissolved in dichloromethane $(20 \mathrm{ml})$, washed with $\mathrm{NaOH} 10 \%$ solution $(2 \times 10 \mathrm{ml})$, and with water $(10 \mathrm{ml})$. Finally, the organic layer was dried $\left(\mathrm{Mg}_{2} \mathrm{SO}_{4}\right)$, concentrated and the residue triturated with hydrobromic acid to obtain the cited compound as hydrobromide $(2 \mathrm{~g}, 50 \mathrm{~g})$, mp $>260^{\circ} \mathrm{C}$ (white microprisms, from ethano1); ${ }^{1} \mathrm{H}-\mathrm{nmr}\left(\mathrm{DMSO}_{6}\right): 2.78\left(3 \mathrm{H}, \mathrm{s}, 1-\mathrm{CH}_{3}\right) ; 4.0-4.2(2 \mathrm{H}, \mathrm{m}, 4-\mathrm{H})$; $4.4-4.6(2 \mathrm{H}, \mathrm{m}, 3-\mathrm{H}) ; 7.2-7.9(5 \mathrm{H}, \mathrm{m})$. (Found: $\mathrm{C}, 50.7 ; \mathrm{H}, 4.75 ; \mathrm{N}, 9.7$. $\mathrm{C}_{12} \mathrm{H}_{13} \mathrm{BrN}_{2} \cdot \mathrm{H}_{2} \mathrm{O}$ requires $\left.\mathrm{C}, 50.9 ; \mathrm{H}, 4.6 ; \mathrm{N}, 9.98\right)$.

\section{Preparation of salts 2-8.}

A. - Quaternization with ethyl bromoacetate. (Compounds 2-4, Table 1). Equivalent amounts (10 mmol) of the corresponding heterocyclic precursor and ethyl bromoacetate in dry acetone $(30 \mathrm{ml})$ were refluxed for the time indicated in Table 1 . The precipitate was filtered off and recrystallized from ethanol-ether.

2: mp 120-122ec (white needles). Ir $1741(\mathrm{C}=0)$, and $1632\left(\mathrm{C}=\mathrm{N}^{+}\right) \mathrm{cm}^{-1}$; $1_{\mathrm{H}-\mathrm{nmr}}\left(\mathrm{DMSO}-\mathrm{d}_{6}\right): 1.32\left(3 \mathrm{H}, \mathrm{t}, \mathrm{J}=7.1 \mathrm{~Hz},-\mathrm{CH}_{2} \mathrm{CH}_{3}\right) ; 2,76\left(3 \mathrm{H}, \mathrm{s}, 1-\mathrm{CH}_{3}\right)$; $4.28\left(2 \mathrm{H}, \mathrm{q}, \mathrm{J}=7.1 \mathrm{~Hz},-\mathrm{COCH}_{2}\right) ; 4.43(2 \mathrm{H}, \mathrm{t}, \mathrm{J}=5.7 \mathrm{~Hz}, 4-\mathrm{H}) ; 4.70$ (2 H, t, $\mathrm{J}=5.7 \mathrm{~Hz}, 3-\mathrm{H}) ; 5.10\left(2 \mathrm{H}, \mathrm{s}, \mathrm{N}^{+} \mathrm{CH}_{2}\right) ; 6.55\left(1 \mathrm{H}, \mathrm{dd}, \mathrm{J}_{7}-6=4.5\right.$ and $\left.\mathrm{J}_{7-8}=2.3 \mathrm{~Hz}, 7-\mathrm{H}\right) ; 7.36\left(1 \mathrm{H}, \mathrm{dd}, \mathrm{J}_{6-7}=4.5\right.$ and $\left.\mathrm{J}_{6-8}=1.3 \mathrm{~Hz}, 6-\mathrm{H}\right) ; 7.66$ (1 $\mathrm{H}$, bs, $8-\mathrm{H}$ ). (Found: C, $46.0 ; \mathrm{H}, 5.8 ; \mathrm{N}, 8.8 . \mathrm{C}_{12} \mathrm{H}_{17} \mathrm{BrN}_{2} \mathrm{O}_{2} .1 / 2 \mathrm{H}_{2} \mathrm{O}$ requires C, $46.4 ; \mathrm{H}, 5.5 ; \mathrm{N}, 9.0 \%)$.

3: mp 187-189 ${ }^{\circ} \mathrm{C}$ (yellow microneedles). Ir $1741(\mathrm{C}=0)$, and $1647\left(\mathrm{C}=\mathrm{N}^{+}\right.$) $\mathrm{cm}^{-1} ;{ }_{\mathrm{H}-\mathrm{nmr}}\left(\mathrm{DMSO}-\mathrm{d}_{6}\right): 1.26\left(3 \mathrm{H}, t, J=7.0 \mathrm{~Hz},-\mathrm{CH}_{2} \mathrm{CH}_{3}\right), 2.96$ (3 H, $s$, $\left.3 \mathrm{H}, 1-\mathrm{CH}_{3}\right) ; 4.24\left(2 \mathrm{H}, \mathrm{q}, \mathrm{J}=7.0 \mathrm{~Hz},-\mathrm{COCH}_{2}\right) ; 5.48\left(2 \mathrm{H}, \mathrm{s}, \mathrm{N}^{+} \mathrm{CH}_{2}\right) ; 7.35-$ $7.4(1 \mathrm{H}, \mathrm{m}, 7-\mathrm{H}) ; 7.75(1 \mathrm{H}, \mathrm{d}, \mathrm{J}=5.9 \mathrm{~Hz}, 4-\mathrm{H}) ; 7.9-8.0(1 \mathrm{H}, \mathrm{m}, 6-\mathrm{H})$; $8.43(1 \mathrm{H}, \mathrm{bs}, 8-\mathrm{H}) ; 8.73(1 \mathrm{H}, \mathrm{d}, \mathrm{J}=5.9 \mathrm{~Hz}, 3-\mathrm{H})$. (Found: $\mathrm{C}, 43.9 ; \mathrm{H}$, $5.2 ; \mathrm{N}, 9.1 . \mathrm{C}_{12} \mathrm{H}_{15} \mathrm{BrN}_{2} \mathrm{O}_{2}$ requires $\left.\mathrm{C}, 44.2 ; \mathrm{H}, 5.05 ; \mathrm{N}, 9.48\right)$.

4: mp 232-234 ${ }^{\circ} \mathrm{C}$ (brown microprisms) Ir $1690(\mathrm{C}=0)$, and $1599\left(\mathrm{C}=\mathrm{N}^{+}\right) \mathrm{cm}^{-1}$; $1_{\mathrm{H}-\mathrm{nmr}}\left(\mathrm{DMSO}-\mathrm{d}_{6}\right): 1.28\left(3 \mathrm{H}, \mathrm{t}, \mathrm{J}=7.0 \mathrm{~Hz},-\mathrm{CH}_{2} \mathrm{CH}_{3}\right) ; 2,87\left(3 \mathrm{H}, \mathrm{s}, 1-\mathrm{CH}_{3}\right)$; $4.27\left(2 \mathrm{H}, \mathrm{q}, \mathrm{J}=7.0 \mathrm{~Hz},-\mathrm{COCH}_{2}\right) ; 4.3-4.4(2 \mathrm{H}, \mathrm{m}, 4-\mathrm{H}) ; 4.5-4.6(2 \mathrm{H}, \mathrm{t}$, $\mathrm{J}=6.9 \mathrm{~Hz}, 3-\mathrm{H}) 5.13\left(2 \mathrm{H}, \mathrm{s}, \mathrm{N}^{+} \mathrm{CH}_{2}\right) ; 7.25-7.3(1 \mathrm{H}, \mathrm{m}, 8-\mathrm{H}) ; 7.55-7.6$ (1 H, m, 7-H); $7.69\left(1 \mathrm{H}, \mathrm{d}, \mathrm{J}_{6-7}=8.6 \mathrm{~Hz}, 6-\mathrm{H}\right) ; 7.85$ (1 H, d, Jg-8 $=7.6 \mathrm{~Hz}$, 
$9-\mathrm{H}) ; 8.13(1 \mathrm{H}, \mathrm{s}, 10-\mathrm{H})$. (Found: C, 54.45; H,5.7; N, 7.75. $\mathrm{C}_{16} \mathrm{H}_{19} \mathrm{BrN}_{2} \mathrm{O}_{2}$ requires $\mathrm{C}, 54.7 ; \mathrm{H}, 5.45 ; \mathrm{N}, 8.08)$.

B.- Amination with MSH. (Compounds 5-8, Table 1).

To a stirred solution of 0-hydroxylaminomesitylenesulfonate (MSH) (2.15 g, $10 \mathrm{mmol})$ in dichloromethane $(20 \mathrm{ml})$, the corresponding azine $(10 \mathrm{mmol})$ in the same solvent $(20 \mathrm{ml})$ was dropwise added. The mixture was stirred at room temperature for $10 \mathrm{~min}$. Diethyl ether $(30 \mathrm{~m} 1)$ was then added to precipitate the $\mathrm{N}$-aminoazinium salts 5-8 which were triturated with ether $(3 \times 5 \mathrm{ml})$ and recrystallized from the suitable solvent.

5: mp 145-147\% (white prisms, from ethanol-ether). Ir $1620\left(\mathrm{C}=\mathrm{N}^{+}\right.$) $\mathrm{cm}^{-1}$; $1_{\mathrm{H}-\mathrm{nmr}}\left(\mathrm{DMSO}-\mathrm{d}_{6}\right): 2.85\left(3 \mathrm{H}, \mathrm{s}, 1-\mathrm{CH}_{3}\right) ; 4.4-4.7(4 \mathrm{H}, \mathrm{m}, 3-\mathrm{H}$ and $4-\mathrm{H})$; 6.65-6.7 (1 H, m, 7-H); 7.2-7.25 (1 H, m, 8-H); 7.6-7.65 (1 H, m, 6-H). (Found: C, $56.5 ; \mathrm{H}, 6.6 ; \mathrm{N}, 12.0 . \mathrm{C}_{17} \mathrm{H}_{23} \mathrm{~N}_{3} \mathrm{O}_{3} \mathrm{~S} .1 / 2 \mathrm{H}_{2} \mathrm{O}$ requires C, $56.9 ; \mathrm{H}$, $6.5 \mathrm{~N}, 11.78)$.

6: mp 147-148.C (white prisms, from ethanol-ether). Ir $1635\left(\mathrm{C}=\mathrm{N}^{+}\right) \mathrm{cm}^{-1}$; $1_{\mathrm{H}-\mathrm{nmr}}\left(\mathrm{DMSO}-\mathrm{d}_{6}\right): 2.95\left(3 \mathrm{H}, 1-\mathrm{CH}_{3}\right) ; 7.26\left(1 \mathrm{H}, \mathrm{dd}, \mathrm{J}_{7-6}=4.5\right.$ and $\mathrm{J}_{7-8}=2.3$ $\mathrm{Hz}, 7-\mathrm{H}) ; 7.54(1 \mathrm{H}, \mathrm{d}, \mathrm{J}=5.9 \mathrm{~Hz}, 4-\mathrm{H}) ; 7.64-7.66(1 \mathrm{H}, \mathrm{m}, 6-\mathrm{H}) ; 8.11$ (1H, dd, $\mathrm{J}_{8-7}=2.3$ and $\left.\mathrm{J}_{8-6}=1.2 \mathrm{~Hz}, 8-\mathrm{H}\right) ; 8.44(1 \mathrm{H}, \mathrm{d}, \mathrm{J}=5.8 \mathrm{~Hz}, 3-\mathrm{H})$. (Eound: C, $55.5 ; \mathrm{H}, 6.5 ; \mathrm{N}, 11.6 . \mathrm{C}_{17} \mathrm{H}_{21} \mathrm{~N}_{3} \mathrm{O}_{3} \mathrm{~S}$ requires $\mathrm{C}, 55.9 ; \mathrm{H}, 6.3 ; \mathrm{N}, 11.5$ \%)

7: mp $142-144^{\circ} \mathrm{C}$ (white microprisms, from ethanol-ether). Ir $1643\left(\mathrm{C}=\mathrm{N}^{+}\right.$) $\mathrm{cm}^{-1} ; 1_{\mathrm{H}-\mathrm{nmr}}\left(\mathrm{DMSO}-\mathrm{d}_{6}\right): 2.70\left(3 \mathrm{H}, \mathrm{s}, 1-\mathrm{CH}_{3}\right) ; 4.24(2 \mathrm{H}, \mathrm{q}, \mathrm{J}=6.0 \mathrm{~Hz}, 4-$ H); $4.47(2 \mathrm{H}, \mathrm{q}, \mathrm{J}=6.1 \mathrm{~Hz}, 3-\mathrm{H}) ; 7.3-7.9(5 \mathrm{H}, \mathrm{m})$. (Found: $\mathrm{C}, 63.35$; $\mathrm{H}, 6.5 ; \mathrm{N}, 10.2 . \mathrm{C}_{21} \mathrm{H}_{25} \mathrm{~N}_{3} \mathrm{O}_{3} \mathrm{~S}$ requires $\left.\mathrm{C}, 63.1 ; \mathrm{H}, 6.3 ; \mathrm{N}, 10.58\right)$.

8: mp 224-226 $\mathrm{C}$ (white prisms, from methanol-ether). Ir $1633\left(\mathrm{C}=\mathrm{N}^{+}\right) \mathrm{cm}^{-1}$; $1_{\mathrm{H}-\mathrm{nmr}}\left(\mathrm{DMSO}-\mathrm{d}_{6}\right): 3.13\left(3 \mathrm{H}, \mathrm{s}, 4-\mathrm{CH}_{3}\right) ; 7.35\left(1 \mathrm{H}, \mathrm{dd}, \mathrm{J}_{2-1}=4.4 \mathrm{~Hz}\right.$ and $\left.\mathrm{J}_{2-3}=2.6 \mathrm{~Hz}, 2-\mathrm{H}\right), 7.6-8.0(3 \mathrm{H}, \mathrm{m}), 8.4-8.7(2 \mathrm{H}, \mathrm{m}), 9.04(1 \mathrm{H}$, dd, $\mathrm{J}_{3-2}=2.6$ and $\mathrm{J}_{3-1}=1.2 \mathrm{~Hz}, 3-\mathrm{H}$ ). (Found: C, $60.2 ; \mathrm{H}, 6.2 ; \mathrm{N}, 10.2$. $\mathrm{C}_{21} \mathrm{H}_{25} \mathrm{~N}_{3} \mathrm{O}_{3} \mathrm{~S}$. $\mathrm{H}_{2} \mathrm{O}$ requires $\mathrm{C}, 60.4 ; \mathrm{H}, 6.0 ; \mathrm{N}, 10.08$ ).

Westphal Condensation. General Procedure. Equivalent amounts ( $10 \mathrm{mmol})$ of the azinium salts 2-8, the dicarbonyl derivative, and anhydrous sodium acetate $(0.82 \mathrm{~g}, 10 \mathrm{mmol})$ were suspended in dry acetone $; 10 \mathrm{ml})$. The mixture was refluxed for the time described (Table 1). The precipitate was filtered. Crystallization from the suitable solvent yielded the compounds 9-15 in analytical grade. 
9a: mp 190-192C (yellow needles, from ethanol). Ir $1734(\mathrm{C}=0), 1618 \quad\left(\mathrm{C}=\mathrm{N}^{+}\right.$) $\mathrm{cm}^{-1} ;{ }^{1} \mathrm{H}-\mathrm{nmr}\left(\mathrm{DMSO}-\mathrm{d}_{6}\right): 1.36\left(3 \mathrm{H}, t, J=7.1 \mathrm{~Hz},-\mathrm{CH}_{2} \mathrm{CH}_{3}\right) ; 2.25$ (3. H, s, 9$\left.\mathrm{CH}_{3}\right) ; 2.52\left(3 \mathrm{H}, \mathrm{s}, 10-\mathrm{CH}_{3}\right) ; 4.5-4.7\left(6 \mathrm{H}, \mathrm{s}, 3 \mathrm{CH}_{2}\right) ; 6.45(1 \mathrm{H}$, dd, $\mathrm{J}_{2-3}=4.0$ and $\left.\mathrm{J}_{2-1}=2.4 \mathrm{~Hz}, 2-\mathrm{H}\right) ; 7.38\left(1 \mathrm{H}\right.$, dd, $\mathrm{J}_{1-2}=4.0$ and $\mathrm{J}_{1-3}=1.4 \mathrm{~Hz}$, $1-\mathrm{H}) ; 7.42-7.43(1 \mathrm{H}, \mathrm{m}, 3-\mathrm{H}) ; 8.35(1 \mathrm{H}, \mathrm{s}, 11-\mathrm{H}) .{ }^{13} \mathrm{C}-\mathrm{nmr}\left(\mathrm{DMSO}-\mathrm{d}_{6}\right)$. Uv $(378,3.93),(308,3.60),(293,3.58),(202.5,4.08)$ (Found: C, 51.1; $\mathrm{H}, 5.15 ; \mathrm{N}, 7.1 . \mathrm{C}_{16} \mathrm{H}_{19} \mathrm{BrN}_{2} \mathrm{O}_{2} .1 / 2 \mathrm{H}_{2} \mathrm{O}$ requires $\left.\mathrm{C}, 50.8 ; \mathrm{H}, 5.1 ; \mathrm{N}, 7.4 \%\right)$.

9b: mp 197-200C (brown prisms, from ethanol). Ir $1741(\mathrm{C}=0), 1632\left(\mathrm{C}=\mathrm{N}^{+}\right.$) $\mathrm{cm}^{-1} ;{ }_{\mathrm{H}-\mathrm{nmr}}($ DMSO-d $): 1.57\left(3 \mathrm{H}, \mathrm{t}, \mathrm{J}=7.1 \mathrm{~Hz},-\mathrm{CH}_{2} \mathrm{CH}_{3}\right) ; 4.6-4.7(2 \mathrm{H}, \mathrm{m}$, $5-\mathrm{H}) ; 4.8-5.0\left(4 \mathrm{H}, \mathrm{m}, \mathrm{COCH}_{2}\right.$ and $\left.6-\mathrm{H}\right) ; 6.57\left(1 \mathrm{H}, \mathrm{dd}, \mathrm{J}_{2}-3=4.1\right.$ and $\mathrm{J}_{2-1}=$ $2.1 \mathrm{~Hz}, 2-\mathrm{H}) ; 7.39(1 \mathrm{H}$, bs, $1-\mathrm{H}) ; 7.57\left(1 \mathrm{H}, \mathrm{dd}, \mathrm{J}_{3-2}=4.0\right.$ and $\mathrm{J}_{3-1}=1.2$ $\mathrm{Hz}, 3-\mathrm{H}) ; 7.8-8.2(4 \mathrm{H}, \mathrm{m}) ; 8.31(1 \mathrm{H}, \mathrm{d}, \mathrm{J}=8.2 \mathrm{~Hz}) ; 8.55(1 \mathrm{H}, \mathrm{d}, \mathrm{J}=7.1$ $\mathrm{Hz}) ; 8.87(1 \mathrm{H}, \mathrm{s}, 11-\mathrm{H})$. Uv $(470,4.43),(455.5,4.43),(343,4.43)$, $(298,4.42),(243,4.42),(218,4.40)$. (Found: C, 59.8; H, 4.4; N, 5.8. $\mathrm{C}_{24} \mathrm{H}_{19} \mathrm{BrN}_{2} \mathrm{O}_{2} . \mathrm{H}_{2} \mathrm{O}$ : requires $\mathrm{C}, 61.9 ; \mathrm{H}, 4.2 ; \mathrm{N}, 6.08$ ).

9c: $\mathrm{mp}>250^{\circ} \mathrm{C}$ (brown microprisms, from ethanol). Ir $1731(\mathrm{C}=0), 1612$ $\left(\mathrm{C}=\mathrm{N}^{+}\right) \mathrm{cm}^{-1} ;{ }_{\mathrm{H}-\mathrm{nmr}}\left(\mathrm{DMSO}-\mathrm{d}_{6}\right): 1.41\left(3 \mathrm{H}, \mathrm{t}, \mathrm{J}=7.1 \mathrm{~Hz},-\mathrm{CH}_{2} \mathrm{CH}_{3}\right) ; 4.5-4.7$ $\left(6 \mathrm{H}, \mathrm{m}, 3 \mathrm{CH}_{2}\right) ; 6.54\left(1 \mathrm{H}, \mathrm{dd}, \mathrm{J}_{2-3}=4.1\right.$ and $\left.\mathrm{J}_{2-1}=2.5 \mathrm{~Hz}, 2-\mathrm{H}\right) ; 7.33$ (1 $\mathrm{H}$, dd, $\mathrm{J}_{3-2}=2.5$ and $\left.\mathrm{J}_{3-1}=1.3 \mathrm{~Hz}, 3-\mathrm{H}\right) ; 7.6-7.9(5 \mathrm{H}, \mathrm{m}) ; 8.1-8.2(1 \mathrm{H}, \mathrm{m})$; $8.6-8.8(2 \mathrm{H}, \mathrm{m}) ; 9.08(1 \mathrm{H}, \mathrm{s}, 11-\mathrm{H})$. (Found: $\mathrm{C}, 63.5 ; \mathrm{H}, 4.7 ; \mathrm{N}, 5.4$. $\mathrm{C}_{26} \mathrm{H}_{21} \mathrm{BrN}_{2} \mathrm{O}_{2} \cdot \mathrm{H}_{2} \mathrm{O}$ requires $\mathrm{C}, 63.55 ; \mathrm{H}, 4.3 ; \mathrm{N}, 5.2$

10a: $\mathrm{mp}>250^{\circ} \mathrm{C}$ (yellow needles, from ethanol). Ir $1733(\mathrm{C}=0), 1628\left(\mathrm{C}=\mathrm{N}^{+}\right.$) $\mathrm{cm}^{-1} ;{ }_{\mathrm{H}-\mathrm{nmr}}\left(\mathrm{DMSO}-\mathrm{d}_{6}\right): 1.49\left(3 \mathrm{H}, \mathrm{t}, \mathrm{J}=7.0 \mathrm{~Hz},-\mathrm{CH}_{2} \mathrm{CH}_{3}\right) ; 2.44$ (3 H, s, 9$\left.\mathrm{CH}_{3}\right) ; 2.68\left(3 \mathrm{H}, \mathrm{s}, 1-\mathrm{CH}_{3}\right) ; 4.73\left(2 \mathrm{H}, \mathrm{q}, \mathrm{J}=7.0 \mathrm{~Hz},-\mathrm{CH}_{2} \mathrm{CH}_{3}\right) ; 7.1-7.2$ (1 $\mathrm{H}, \mathrm{m}, 2-\mathrm{H}) ; 7.77(1 \mathrm{H}, \mathrm{d}, \mathrm{J}=6.1 \mathrm{~Hz}, 5-\mathrm{H}) ; 7.87\left(1 \mathrm{H}, \mathrm{d}, \mathrm{J}_{3-2}=4.1 \mathrm{~Hz}, 3-\mathrm{H}\right)$; $8.0-8.05(1 \mathrm{H}, \mathrm{bs}, 1-\mathrm{H}) ; 8.50(1 \mathrm{H}, \mathrm{d}, \mathrm{J}=6.1 \mathrm{~Hz}, 6-\mathrm{H}) ; 8.63(1 \mathrm{H}, \mathrm{s}, 11-\mathrm{H})$. (Found: $\mathrm{C}, 52.3 ; \mathrm{H}, 4.8 ; \mathrm{N}, 8.0 . \mathrm{C}_{16} \mathrm{H}_{17} \mathrm{BrN}_{2} \mathrm{O}_{2} . \mathrm{H}_{2} \mathrm{O}$ requires $\mathrm{C}, 52.3 ; \mathrm{H}$, $4.7 ; \mathrm{N}, 7.6 \%$.

10b: mp 223-225ㄷ (brown prisms, from ethanol-ether). Ir 1731 (C=0), 1645 $\left(\mathrm{C}=\mathrm{N}^{+}\right) \mathrm{cm}^{-1} ;{ }^{1} \mathrm{H}-\mathrm{nmr}\left(\mathrm{DMSO}-\mathrm{d}_{6}\right): 1.58\left(3 \mathrm{H}, \mathrm{t}, \mathrm{J}=7.1 \mathrm{~Hz},-\mathrm{CH}_{2} \mathrm{CH}_{3}\right) ; 4.90$ (2 $\left.\mathrm{H}, \mathrm{q}, \mathrm{J}=7.1 \mathrm{~Hz},-\mathrm{CH}_{2} \mathrm{CH}_{3}\right) ; 7.24\left(1 \mathrm{H}\right.$, dd, $\mathrm{J}_{2-3}=4.5$ and $\left.\mathrm{J}_{2-1}=2.5 \mathrm{~Hz}, 2-\mathrm{H}\right)$; $7.5-8.6(10 \mathrm{H}, \mathrm{m}) ; 9.29(1 \mathrm{H}, \mathrm{s}, 11-\mathrm{H})$. Uv $(450,4.13),(412.5,4.16)$, $(348,4.31),(341,4.29),(305,4.31),(243,4.46),(225,4.44)$. (Found: C, $62.3 ; \mathrm{H}, 3.4 ; \mathrm{N}, 5.8 . \mathrm{C}_{24} \mathrm{H}_{1} 7 \mathrm{BrN}_{2} \mathrm{O}_{2} . \mathrm{H}_{2} \mathrm{O}$ requires $\mathrm{C}, 62.2 ; \mathrm{H}, 3.7 ; \mathrm{N}$, $6.058)$. 
11a: $\mathrm{mp}>250^{\circ} \mathrm{C}$ (yellow needles, from ethanol). Ir $1733(\mathrm{C}=0), 1638\left(\mathrm{C}=\mathrm{N}^{+}\right.$) $\mathrm{cm}^{-1} ;{ }^{1} \mathrm{H}-\mathrm{nmr}\left(\mathrm{CD}_{3} \mathrm{OD}\right): 1.50\left(3 \mathrm{H}, t, \mathrm{~J}=7.1 \mathrm{~Hz},-\mathrm{CH}_{2} \mathrm{CH}_{3}\right) ; 2.41(3 \mathrm{H}, \mathrm{s}, 11-$ $\left.\mathrm{CH}_{3}\right) ; 2.66\left(3 \mathrm{H}, \mathrm{s}, 12-\mathrm{CH}_{3}\right) ; 4.6-4.7\left(4 \mathrm{H}, \mathrm{m}, 7-\mathrm{H}\right.$ and $\left.-\mathrm{CH}_{2} \mathrm{CH}_{3}\right) ; 4.8-4.9$ (2 $\mathrm{H}, \mathrm{m}, 8-\mathrm{H}) ; 7.2-7.25(1 \mathrm{H}, \mathrm{m}, 3-\mathrm{H}) ; 7.4-7.45(1 \mathrm{H}, \mathrm{m}, 4-\mathrm{H}) ; 7.69$ (1 H, d, $\left.J_{5-4}=8.6 \mathrm{~Hz}, 5-\mathrm{H}\right) ; 7.85\left(1 \mathrm{H}, \mathrm{d}, \mathrm{J}_{2-3}=7.6 \mathrm{~Hz}, 2-\mathrm{H}\right) ; 8.49$ (1 H, s, 13-H) . Uv $(389.5,4.32),(235,4.15),(219.5,4.33)$. (Found: C, 61.3; H, 5.0; N, 6.7. $\mathrm{C}_{20} \mathrm{H}_{21} \mathrm{BrN}_{2} \mathrm{O}_{2}$ requires $\mathrm{C}, 61.0 ; \mathrm{H}, 5.1 ; \mathrm{N}, 6.8 \%$ ).

11b: $\mathrm{mp}>250^{\circ} \mathrm{C}$ (brown needles, ethanol-ether). Ir $1737(\mathrm{C}=0), 1616\left(\mathrm{C}=\mathrm{N}^{+}\right.$) $\mathrm{cm}^{-1} ;{ }^{1} \mathrm{H}-\mathrm{nmr}\left(\mathrm{CD}_{3} \mathrm{OD}\right): 1.58\left(3 \mathrm{H}, \mathrm{t}, \mathrm{J}=7.1 \mathrm{~Hz},-\mathrm{CH}_{2} \mathrm{CH}_{3}\right) ; 4.7-4.9$ (4 H, m, $7-\mathrm{H}$ and $\left.-\mathrm{CH}_{2} \mathrm{CH}_{3}\right) ; 5.1-5.2(2 \mathrm{H}, \mathrm{m}, 8-\mathrm{H}) ; 7.2-7.3(1 \mathrm{H}, \mathrm{m}, 3-\mathrm{H}) ; 7.4-7.5$ (1 H, m, 4-H); $7.62\left(1 \mathrm{H}, \mathrm{d}, \mathrm{J}_{5-4}=8.1 \mathrm{~Hz}, 5-\mathrm{H}\right) ; 7.82\left(1 \mathrm{H}, \mathrm{d}, \mathrm{J}_{2-3}=7.7 \mathrm{~Hz}\right.$, $2-\mathrm{H}) ; 7.9-8.2(4 \mathrm{H}, \mathrm{m}) ; 8.3-8.5(2 \mathrm{H}, \mathrm{m}) ; 8.7-8.8(1 \mathrm{H}, \mathrm{m}) ; 9.37(1 \mathrm{H}, \mathrm{s}$, $13-\mathrm{H})$. Uv $(469,4.08),(318,3.96),(251.5,4.13),(212,4.41)$. (Found: C, $67.35 ; \mathrm{H}, 4.3 ; \mathrm{N}, 5.8 . \mathrm{C}_{28} \mathrm{H}_{21} \mathrm{BrN}_{2} \mathrm{O}_{2}$ requires $\left.\mathrm{C}, 67.6 ; \mathrm{H}, 4.3 ; \mathrm{N}, 5.68\right)$.

12b: $\mathrm{mp}>250^{\circ} \mathrm{C}$ (brown prisms, from ethanol-ether). Ir $1627\left(\mathrm{C}=\mathrm{N}^{+}\right) \mathrm{cm}^{-1}$; $1_{\mathrm{H}-\mathrm{nmr}}\left(\mathrm{CD}_{3} \mathrm{OD}\right): 4.75(2 \mathrm{H}, \mathrm{t}, \mathrm{J}=7.3 \mathrm{~Hz}, 5-\mathrm{H}) ; 5.16(2 \mathrm{H}, \mathrm{t}, \mathrm{J}=7.3 \mathrm{~Hz}, 6-$ H); $6.62\left(1 \mathrm{H}, \mathrm{dd}, \mathrm{J}_{2-3}=4.1 \mathrm{~Hz}\right.$ and $\left.\mathrm{J}_{2-1}=2.3 \mathrm{~Hz}, 2-\mathrm{H}\right) ; 7.47$ (1 H, bs, $\left.1-\mathrm{H}\right)$; $7.6-7.7(1 \mathrm{H}, \mathrm{m}, 3-\mathrm{H}) ; 8.0-8.1(2 \mathrm{H}, \mathrm{m}) ; 8.4-8.7(4 \mathrm{H}, \mathrm{m}) ; 9.15(1 \mathrm{H}, \mathrm{s}, 11$ H). Uv $(450,4.40),(439,4.40),(346,4.27),(298,4.405),(275,4.375)$, (233.5, 4.43) (Found: C, $68.8 ; \mathrm{H}, 5.4 ; \mathrm{N}, 8.5 . \mathrm{C}_{29} \mathrm{H}_{25} \mathrm{~N}_{3} \mathrm{O}_{3} \mathrm{~S} .1 / 2 \mathrm{H}_{2} \mathrm{O}$ requires $\mathrm{C}, 69.0 ; \mathrm{H}, 5.0 ; \mathrm{N}, 8.38)$.

12c: $\mathrm{mp}>250^{\circ} \mathrm{C}$ (brown prisms, from ethanol-ether). Ir $1615\left(\mathrm{C}=\mathrm{N}^{+}\right) \mathrm{cm}^{-1}$; ${ }^{1} \mathrm{H}-\mathrm{nmr}\left(\mathrm{CD}_{3} \mathrm{OD}\right): 4.82(2 \mathrm{H}, t, J=6.7 \mathrm{~Hz}, 5-\mathrm{H}): 5.29(2 \mathrm{H}, t, J=6.7 \mathrm{~Hz}, 6-$ H); $6.59\left(1 \mathrm{H}\right.$, dd, $\mathrm{J}_{2-3}=4.1$ and $\left.\mathrm{J}_{2-1}=2.3 \mathrm{~Hz}, 2-\mathrm{H}\right) ; 7.42(1 \mathrm{H}, \mathrm{bs}, 1-\mathrm{H})$; $7.7-8.0(5 \mathrm{H}, \mathrm{m}) ; 8.6-8.7(2 \mathrm{H}, \mathrm{m}) ; 8.9-9.0(2 \mathrm{H}, \mathrm{m}) ; 9.50(1 \mathrm{H}, \mathrm{s}, 11 \mathrm{H})$. Uv $(439,3.855),(362.5,4.35),(340,4.50),(253.5,4.35),(223.5,4.47)$ (Found: $\mathrm{C}, 68.2 ; \mathrm{H}, 5.3 ; \mathrm{N}, 7.5 . \mathrm{C}_{31} \mathrm{H}_{27} \mathrm{~N}_{3} \mathrm{O}_{3} \mathrm{~S} .1 / 2 \mathrm{H}_{2} \mathrm{O}$ requires $\mathrm{C}, 67.9 ; \mathrm{H}$, $5.0 ; \mathrm{N}, 7.78)$.

13b: mp $>250^{\circ} \mathrm{C}$ (brown prisms, from ethanol). Ir $1627\left(\mathrm{C}=\mathrm{N}^{+}\right.$) $\mathrm{cm}^{-1} ; 1_{\mathrm{H}-\mathrm{nmr}}$ $\left(\mathrm{CD}_{3} \mathrm{OD}\right)=7.35\left(1 \mathrm{H}, \mathrm{dd}, \mathrm{J}_{2-3}=4.0\right.$ and $\left.\mathrm{J}_{2-1}=2.6 \mathrm{~Hz}\right) ; 7.9-8.0(2 \mathrm{H}, \mathrm{m}) ; 8.0-$ $8.1(1 \mathrm{H}, \mathrm{m}, 3-\mathrm{H}) ; 8.19-8.21(1 \mathrm{H}, \mathrm{m}, 1-\mathrm{H}) ; 8.3-8.7(6 \mathrm{H}, \mathrm{m}) ; 9.48$ (1 H, $s, 11-H)$. Uv $(412.5,4.39),(380,4.39),(353.5,4.51),(300,4.405)$, (242.5, 4.51). (Found: C, $67.2 ; \mathrm{H}, 4.8 ; \mathrm{N}, 8.3 . \mathrm{C}_{29} \mathrm{H}_{23} \mathrm{~N}_{3} \mathrm{O}_{3} \mathrm{~S} .1 / 2 \mathrm{H}_{2} \mathrm{O}$ : requires $\mathrm{C}, 66.9 ; \mathrm{H}, 4.45 ; \mathrm{N}, 8.18)$.

14b: $\mathrm{mp}>250^{\circ} \mathrm{C}$ (brown prisms, from ethanol). Ir $1624\left(\mathrm{C}=\mathrm{N}^{+}\right) \mathrm{cm}^{-1} ;{ }^{1} \mathrm{H}-\mathrm{nmr}$ 
$\left(\mathrm{CD}_{3} \mathrm{OD}\right): 4.8-4.9(2 \mathrm{H}, \mathrm{m}, 7-\mathrm{H}) ; 5.3-5.4(2 \mathrm{H}, \mathrm{m}, 8-\mathrm{H}) ; 7.2-7.3(1 \mathrm{H}, \mathrm{m}$, $3-\mathrm{H}) ; 7.4-7.5(1 \mathrm{H}, \mathrm{m}, 4-\mathrm{H}) ; 7.66\left(1 \mathrm{H}, \mathrm{d}, \mathrm{J}_{5-4}=8.3 \mathrm{~Hz}, 5-\mathrm{H}\right) ; 7.83$ (1 H, d, $\left.J_{2-3}=7.9 \mathrm{~Hz}, 2-\mathrm{H}\right) ; 8.0-8.15(2 \mathrm{H}, \mathrm{m}) ; 8.5-8.7(4 \mathrm{H}, \mathrm{m}) ; 9.50(1 \mathrm{H}, \mathrm{s}$, $13-H)$. Uv $(452,4.14),(309,4.01),(273,3.92),(246,4.03),(215.5$, 4.44). (Found: $\mathrm{C}, 71.3 ; \mathrm{H}, 5.0 ; \mathrm{N}, 7.4 . \mathrm{C}_{33} \mathrm{H}_{27} \mathrm{~N}_{3} \mathrm{O}_{3} \mathrm{~S} .1 / 2 \mathrm{H}_{2} \mathrm{O}$ requires $\mathrm{C}$, $71.5 ; \mathrm{H}, 4.9 ; \mathrm{N}, 7.6 \%)$

14c: $\mathrm{mp}>250^{\circ} \mathrm{C}$ (brown needles, from ethanol). Ir $1624\left(\mathrm{C}=\mathrm{N}^{+}\right) \mathrm{cm}^{-1} ;{ }^{1} \mathrm{H}-\mathrm{nmr}$ $\left(\mathrm{CD}_{3} \mathrm{OD}\right): 4.9-5.0(2 \mathrm{H}, \mathrm{m}, 7-\mathrm{H}) ; 5.5-5.6(2 \mathrm{H}, \mathrm{m}, 8-\mathrm{H}) ; 7.2-7.3(1 \mathrm{H}, \mathrm{m}$, $3-\mathrm{H}) ; 7.45-7.55(1 \mathrm{H}, \mathrm{m}, 4-\mathrm{H}) ; 7.66\left(1 \mathrm{H}, \mathrm{d}, \mathrm{J}_{2-3}=7.7 \mathrm{~Hz}, 2-\mathrm{H}\right) ; 7.8-8.1$ $(6 \mathrm{H}, \mathrm{m}) ; 8.8-8.9(2 \mathrm{H}, \mathrm{m}) ; 9.1-9.2(2 \mathrm{H}, \mathrm{m}) ; 9.93(1 \mathrm{H}, \mathrm{s}, 13-\mathrm{H})$. Uv $(371,4.37),(353,4.47),(257,4.40),(232.5,4.51)$. (Found: C, 71.5; H, 5.1; N, 7.3. $\mathrm{C}_{35} \mathrm{H}_{29} \mathrm{~N}_{3} \mathrm{O}_{3} \mathrm{~S} . \mathrm{H}_{2} \mathrm{O}$ requires $\left.\mathrm{C}, 71.3 ; \mathrm{H}, 5.0 ; \mathrm{N}, 7.1 \frac{8}{8}\right)$.

15b: mp $>250^{\circ} \mathrm{C}$ (brown prisms, from ethanol). Ir $1631\left(\mathrm{C}=\mathrm{N}^{+}\right) \mathrm{cm}^{-1} ; 1_{\mathrm{H}-\mathrm{nmr}}$ $\left(C_{3} \mathrm{OD}\right)=7.24\left(1 \mathrm{H}\right.$, dd, $\mathrm{J}_{2-3}=4.3$ and $\left.\mathrm{J}_{2-1}=2.7 \mathrm{~Hz}\right) ; 7.7-7.9(4 \mathrm{H}, \mathrm{m}) ; 8.04$ $(1 \mathrm{H}, \mathrm{bd}, 3-\mathrm{H}) ; 8.1-8.5(5 \mathrm{H}, \mathrm{m}) ; 8.65(1 \mathrm{H}, \mathrm{bd}, 1-\mathrm{H}) ; 8.9-9.0(1 \mathrm{H}, \mathrm{m})$; $9.40(1 \mathrm{H}, \mathrm{s}, 11-\mathrm{H})$ (Found: $\mathrm{C}, 70.3 ; \mathrm{H}, 5.0 ; \mathrm{N}, 7.3 . \mathrm{C}_{33} \mathrm{H}_{27} \mathrm{~N}_{3} \mathrm{O}_{3} \mathrm{~S} . \mathrm{H}_{2} \mathrm{O}$ requires $\left.\mathrm{C}, 70.3 ; \mathrm{H}, 4.8 ; \mathrm{N}, 7.5 \frac{8}{8}\right)$.

15c: $\mathrm{mp}>250^{\circ} \mathrm{C}$ (brown prisms, from ethanol-ether). Ir $1639\left(\mathrm{C}=\mathrm{N}^{+}\right) \mathrm{cm}^{-1}$; $1_{\mathrm{H}-\mathrm{nmr}}\left(\mathrm{CD}_{3} \mathrm{OD}\right): 7.20-7.25(1 \mathrm{H}, \mathrm{m}, 2-\mathrm{H}) ; 7.5-7.7(5 \mathrm{H}, \mathrm{m}) ; 7.85-7.95(1 \mathrm{H}$, $\mathrm{m}) ; 8.10(1 \mathrm{H}, \mathrm{bd}, 3-\mathrm{H}) ; 8.2-8.3(3 \mathrm{H}, \mathrm{m}) ; 8.4-8.6(3 \mathrm{H}, \mathrm{m}) ; 8.69(1 \mathrm{H}$, bd, $1-\mathrm{H}) ; 9.47(1 \mathrm{H}, \mathrm{s}, 11-\mathrm{H})$ (Found: $\mathrm{C}, 72.7 ; \mathrm{H}, 5.2 ; \mathrm{N}, 7.0$. $\mathrm{C}_{35} \mathrm{H}_{2}{ }_{9} \mathrm{~N}_{3} \mathrm{O}_{3} \mathrm{~S}$. $1 / 2 \mathrm{H}_{2} \mathrm{O}$ requires $\mathrm{C}, 72.4 ; \mathrm{H}, 5.0 ; \mathrm{N}, 7.28$ )

\section{ACKNOWLEDGEMENTS}

Authors wish to acknowledge the assistance provided by LILLY S.A. in Nmr analysis, and to the Comision Interministerial de Ciencia y Tecnologia (CICYT) for financial support (Project PB87-0755).

\section{REFERENCES .}

1. Matoba, K.; Shibata M.; Yamazaki, T. Chem. Pharm. Bull. 1982, 30, 1718 .

2. Alvarez-Builla, J.; Gonzalez Trigo, G.; Ezquerra, J.; Fombella, M. J. Heterocyclic Chem. 1985, 22, 681. 
3. Ezquerra J.; Alvarez-Builla, J. J. Heterocyclic Chem. 1986, 23, 1151.

4. Galera, C.; Vaquero, J. J.; García Navio, J. L.; Alvarez-Builla, J. J. Heterocyclic Chem. 1986, 23, 1889.

5. Pastor, J.; Matia, M. P.; Garcia-Navio, J. L.; Vaquero J. J.; Alvarez-Builla, J. Heterocycles 1989, 29, 2369.

6. Matia, M. P.; Garcia-Navio, J. L.; Vaquero J. J.; Alvarez-Builla, J. J. Heterocyclic Chem. 1990, 27, 661.

7. Westphal, O.; Jahn, K.; Heffe, W. Arch. Pharm. 1961, 294, 37.

8. Jirkovsky I.; Baudy, R. Synthesis 1981, 481 .

9. Flamemt, I.; Sonnay, P.; Ohloff, G. Helv. Chim. Act. 1977, 60, 1872 .

10. Cheeseman G. w. H.; Tuck, B. J. Chem. Soc. (C) $1966,852$.

11. Sánchez-Ferrando F. Magn. Reson. Chem., 1985, 23, 185.

12 Cativiela C.; Sánchez-Ferrando F. Magn. Reson. Chem., 1985, 23, 1072.

13. Pfeil E.; Harder U. Angew. Chem. Int. Ed. Engl, 1967, 6, 178. 
